\title{
The School of Mandarin Duck and Butterfly's Creative Push on Early Chinese Publishing Industry
}

\author{
$\mathrm{Bin} \mathrm{Li}^{1}$ \\ ${ }^{1}$ College of Humanities, Suzhou University of Sciences and Technology, Suzhou, China \\ Correspondence: Bin Li, College of Humanities, Suzhou University of Science and Technology, Ke Rui Road, \\ North area of International Education Park, High-tech Zone of Suzhou, Suzhou 215009, Jiangsu, China. E-mail: \\ xinwenlibin@126.com
}

Received: May 19, 2012 Accepted: June 11, 2012 Online Published: September 20, 2012

doi:10.5539/ass.v8n12p164 URL: http://dx.doi.org/10.5539/ass.v8n12p164

Literati of Jiangsu and Early Chinese Film (2012SJB760039), philosophy and social scientific research fund project of Provincial university of Department of education of Jiangsu.

The School of Mandarin Duck and Butterfly and Early Chinese Culture and Creative Industries (1919-1930) (11YJC760032), humanities and social sciences research project of Ministry of education of China.

The School of Mandarin Duck and Butterfly and Chinese Film Studies from the perspective of communication (XKY201017), social science research project of Suzhou University of Science and Technology.

\begin{abstract}
The School of Mandarin Duck and Butterfly, as an early Chinese popular literature important school, is a participant of early Chinese publishing industry who promoted early Chinese publishing industry development through creativities on publishing from four aspects such as publishing content creativity, graphic design creativity, marketing creativity and cross-media industry creativity.
\end{abstract}

Keywords: the school of mandarin duck and butterfly, publishing industry, creativity

\section{Introduction}

The School of Mandarin Duck and Butterfly is a fresh school of Chinese publishing industry in early stage. Shanghai where their publication creative activities promoted early Chinese publishing industries is a center of Chinese publishing industry in early stage. In 1920-30's, there accommodated occupational workers with certain cultural knowledge from different levels in many commercial mechanism in Shanghai such as millionaire, boss, manager, staff, teacher, student, staff and so on whose cultural life demand in spare time formed a huge spiritual consumption market. The publishing industry flourished corresponding to consumptive demand, called the Golden Age of publishing industry (Liu, 1997). The School of Mandarin Duck and Butterfly is a participant of the early Chinese publishing industry as an early Chinese popular literature important school who promoted early Chinese publishing industry development through creativities on publishing.

This paper firstly studies in depth the history of early Chinese culture and creative industry development and analyzes relationship between the School of Mandarin Duck and Butterfly and early culture creative industry by solid historical data. It also draws a conclusion that the School of Mandarin Duck and Butterfly played an important role in history of early cultural creative industry development through complete, systematic research, which provides some foundation for combing history of Chinese cultural creative industry development and experiences for contemporary cultural and creative industry development. Chinese culture creative industry should not only learn from western development experience but also explore historical experience. Bidirectional extension helps to build a complete, harmonious, plump industrial form and structure. The interaction between the School of Mandarin Duck and Butterfly and early Chinese creative publishing industry is a typical case of early Chinese cultural and creative industries development, which shows that the early Chinese culture and creative industry actively adapted to global economic changes and melted into the world industry structure. The interaction is also an arsenal of experience and spiritual resources for current Chinese culture and creative industry development. The paper also expands research field of cultural and creative industry and breaks through research pattern of traditional popular literature, switches the research perspective from literature modernity to 
modernization of creativity, which applies to research of cultural creative industry with relatively abundant findings of traditional popular literature and lays foundation for the cultural and creative industry development.

\section{Publishing Content Creativity}

Production of content products is a starting point of medium operation and material basis of media brand. The School of Mandarin Duck and Butterfly provided creative content for newspaper at that time, which improved the traditional operation of publishing industry.

\subsection{All Kinds of Novel Creativity}

The School of Mandarin Duck and Butterfly's novels were favored by publications in Shanghai which had been always published by Novel Monthly founded by Commercial press in 1910-1920s as an example. Saturday edited by Zhou Shoujuan was a home of The School of Mandarin Duck and Butterfly's novels. Novel Newspapers edited by Wu Shuangre published Love History with Tears, Remembrance of Lovers, Broken Affectionate Couples by Sticks (by Xu Zhenya), Heartbroken Love, Sweet Memories in Spring and Autumn (by Wu Shuangre), Mr. Pan's Sorrow (by Li Dingyi), Pipa tears (by Jiang Zhu chao), Jade Floor with Spider Net(by Zhu Yuanchu) etc. At the same time there emerged a number of newspapers and periodicals publishing the School of Mandarin Duck and Butterfly's novels such as Red, Happiness, Family, Star Light, Detective World etc. The School of Mandarin Duck and Butterfly also directed and edited various magazines, tabloids and supplements, which amounted to 340 kinds in Shanghai. For example, Novels Pictorial, firstly written with vernacular, was the first vernacular literary journal to some extent, and edited by Bao Tianxiao who participated in editing a total of 18 kinds of newspapers. Besides, his Times's supplement Remaining Interest established an important status of literary supplement, Grand Sight on Novels was the first quarterly of Chinese novel and literary (Nie, 2008:95-98). In addition, Zhou Shoujuan edited Violet, Bao Tianxiao edited week, Zhou Shoujuan and Zhao Caokuang edited Game World which all belonged to journals of the School of Mandarin Duck and Butterfly. There were about a total of 114 species besides tabloids and supplements according to what Zheng Yimei referred in On Old School of Literary Periodicals in Republic of China. With regard to newspaper supplement, Shen Daily "Free Talk", News's Happy Forest, Times's Sideshow Story are amongst a camp of the School of Mandarin Duck and Butterfly. Besides, Crystal, as a representative tabloid, was also a position of popular literature of the School of Mandarin Duck and Butterfly (Zhou, 2005:84-88). The School of Mandarin Duck and Butterfly had intimate contact with many publishing institutions such as World Bookstore, Dadong Bookstore, Civilization Bookstore, China Bookstore, Guangzhi Bookstore, Guohua Bookstore, Qinghua Bookstore, Sanyou Bookstore, Sanxing Bookstore, Shanghai Bookstore, Mass Bookstore, Zhengqi Bookstore, Book\&Magazine Company of China, Civil Rights Press, Qunxue Group, Jinzhang Bookstore, Xian Shuolin Publishing House etc, which mainly published literature books and periodicals of popular literature of the School of Mandarin Duck and Butterfly and had a large number of citizen readers. Taking romantic novels in pursuit of fling, grief at separation and joy as main representative, novels marked with "love", "sorrowful love", "gorgeous love", "tragic love", "complaint love", "regrettable love", "affliction love", "ugly love", "Chivalry love" accounted for 1/4 of total 600 novels published in early 100 periods of Saturday such as Jiang Xingchi's Word Gallbladder and Flute Heart, Mei Lang's Jealous Mind, Chen Xiaodie's Tower Words and Setting Sun, Sweet Flowers and beauty, Wu Shuangre's Dipping in Sesame, Zhou Shoujuan's See You, Forever Regret, Ye Shengtao's Gambler, Tianbai's Tearful Days at White Jade Table, which were constantly followed. Literature works titled Mandarin Duck surged after publication of Jade Pear Soul and broken Swan goose such as Xu Zhenya's Broken Affectionate Couples by Sticks, Chen Diexian's Mandarin Duck Blood, Gong Shaoqin's Mandarin Duck Dream,Li Hanqiu's Mandarin Duck in Evil Sea, Small Printing in Mandarin Duck etc.

\subsection{Development of Creative Publishing Form}

The School of Mandarin Duck and Butterfly had been engaged in the newspaper publishing industry, called the first attempt to participate in cultural industry (Li, 2005:122-125).The School of Mandarin Duck and Butterfly broke through binding constraints and serialized novels, published relying on the newspaper supplement when engaging in publishing industry, such as Zhang Henshui's three representative literature works published in Evening World supplement Luminous Night, New's supplement Happy Forest. Hell on Earth, a total of eighty chapters written by Bi Yihong and Bao Tianxiao, was serialized in Shen Daily Free Talk, popularly accepted, soon led to a booklet(Yin,2009). Zhenya's Jade Pear Soul was serialized in Civil Rights supplement and Su Manshu's Lone Swan Goose was serialized in Pacific Daily supplement. The concept of fractal, nonlinear publishing, recognized by the market, changed traditional publication time and pattern and became a main way of combination between publication industry and publishing industry. Original Story, also known as Movie Novel, is a new reading form created by the School of Mandarin Duck and Butterfly inspired by films. The 
School of Mandarin Duck and Butterfly involved in early creation of Movie Novel such as Zhou Shoujuan's Hero published in Saturday in 1914 , not only describing the eponymous movie' s popularization after being screened ten more nights but also explaining main characters of the movie and equipped with stage photos. The novels had been serialized in the newspapers adapted by Bao Tianxiao from films such as Poor Girl adapted into novel Temptation which had been serialized from October 4 to Sept 31, 1925 in Shen Daily Free Talk, Passionate Actress adapted into novel Kindness and Hatred which had been serialized from January 16 to May 7, 1926 in Shen Daily Free Talk, Rich Girl adapted into novel with same name which had been serialized from May 29 to June 26,1926 in Shen Daily Free Talk, Poor Girl adapted into novel with same name which had been serialized from September 14 to Dec 27,1926 in Shen Daily Free Talk, Resurrection of Conscience adapted into novel with the same name which had been serialized since December 17,1925 in Hangzhou Pictorial, Merry Little Lady adapted into novel Trade of Love which had been serialized from June 27 to August 13,1926 in Shen Daily Free Talk, Actress Revenge adapted into novel Blind Love which had been serialized from December 18,1926 to July 2,1928 in Shen Daily Free Talk. Lu Danan adapted American film Bloody Man and Black Pirates into detective story, popularly accepted (Zhou, 1919). As Tang Zhesheng said, Lu Danan's detective movie adaptation played the transition role in translation of Chinese detective novels from foreign fiction to native creation (Tang, 2007:187-192). The School of Mandarin Duck and Butterfly involved in publishing many new books, film press which they co-founded and edited a large number of as an example. In film presses, the School of Mandarin Duck and Butterfly published a lot of film criticism. Zhou Shoujuan's Film Talks had been serialized in Shen Daily Free Talk which were neither school rational narratives nor words without sense, but integration of knowledge of history, film background, movie-going feelings, performance evaluation with exquisite language full of subjective emotion and changeable technique, transferred new movie information through the movie text analysis, rather than realized the power of the new division through concept of the film criticism, reflected characteristics of spiritual communication. It also used rigorous, scientific description methods such as digital summary and description and inherited characteristics of unity of historical narrative and literary narrative with line drawing on movie history. Film Talks was influenced by Chinese poetry and sketch-like creation and a fairly modern style on movie history (Chen, 2008:60-67).

\section{Binding and Layout Design Creativity}

At the beginning of twentieth Century, the traditional Book and new Book appeared at the same time and many books used Chinese-Western graphic form. Lin Shu's translation novel published by the Commercial Press used the traditional thread-bound form and foreign lace pattern for decoration. The four famous novels in the late Qing Dynasty such as New Novel, Month, Embroidered Portrait Novel, Novel Forest of which covers reflected gradual influence of west on east for Chinese book design even though design and illustration had been not yet completely out of style of traditional Chinese painting and traditional novel illustrations (Zhu, 2007). The Mandarin Duck and Butterfly's novels were quite popular in early years of the Republic of China whose experiences of publication binding design were some worthy reference for current publishing industry even though considered inevitable infant stage in modern art history (Jia, 2005).

\subsection{Cover Creativity}

The School of Mandarin Duck and Butterfly adapted to aesthetic trends of Shanghai modern public and showed fresh new cover creative. New chic cover was used in Violet edited by Zhou Shoujuan. Bao Tianxiao adjusted and innovated cover of the journal according to fashion of the times and readers' psychological needs as well as journal content requirements such as cover of the women's times of every period was portrayed with meticulous ladies and copperplate photography of famous women or students in Ladies's College were published before the formal text involved an academic distinguished personage Lu Bicheng, a female artist Tao Tao, a Su embroidery master Shen Shou etc. The masters such as Dingsong, Du Yu, Xue Ni etc, engaged by special arrangement of Novel Pictorial as editor-in-chief, bound cover with painting of animals such tiger, lion, elephant, peacock, parrot and performed very well(Nie,2008). Novel Times bound the cover with courtesan or actress pictures. A lot of publications of the School of Mandarin Duck and Butterfly began to follow after that, such as Women's World, Game Magazine, Novel Monthly, Eyebrow Talks, Civil Rights, Violet used watercolor beauty picture as cover. Portraits of Women with elegant refined appearance and traditional clothing were often in covers of early 100 periods of Saturday and women with dress, shoe of western style were in the late 100 periods. In the late 100 periods, Zhou Shoujuan slightly changed their style, published poems of a celebrity and invited Dingsong to created portrait according to their poetic meaning, at the same time equipped with some comics, landscapes and pictures suitable for special issue as well as specific year such as the cover of Christmas supplementary issue in 1921 is Santa Claus gifting people with a giant bag full of Saturday. Commemorating the ten anniversary of the establishment of the Republic of China in October 10, 1921, the cover of the thirty special constituted the body 
of pictures composed by three crosses which the pedestrians all looked on and revealed the solemn patriotism and feelings of misery. The inscription in Cover of Saturday was exquisite, beautiful and varied. The famous persons such as Wang Dungen, Ye Zhongling, Wu Zhiying, Zhang Yuguang, Zhang Danfu, Wang Dacuo, Liu Haisu and so on inscribed titles for Saturday in early 100 periods.

\subsection{Materials Creativity}

The School of Mandarin Duck and Butterfly took the lead in using new materials. Copperplate photos were used in Saturday which referred to Chinese and foreign political and cultural celebrities, famous actresses and landscapes etc. Type also changed, such as Novel Pictorial chief-edited by Bao Tianxiao gave up usual stereotype, replaced with glazed paper lithography, bound up with colored silk of Japanese type imitation, which was popular best-selling books around the regions. Grand Sight on Novel chief-edited by Bao Tianxiao used cardboard as cover and invited cultural celebrities to inscribed titles such as Yu Youren inscribing title was stout, straight and antique new fun (Bao, 1971). And then using the color overprint also showed some innovation. There attached numerous illustrations with heart-shaped outline in 115th period of Saturday, which showed love color.

\subsection{Column Creativity}

\subsubsection{Column Refinement}

With entertainment and fun as the center, the column of Saturday was designed to publish mainly sentimental novels classified as Sorrowful Love, Gorgeous Love, Tragic Love, Complaint Love, Regrettable Love, Affliction Love, Ugly Love, Chivalry Love etc. In addition, it also published detective fictions, adventure novels, exploration fictions, fantasy fictions, mystery novels and rogue novels etc. The new novels written by new vernacular and modern punctuation altered the malady of formal monotone and helped readers find content that they hoped to understand so as to make the magazine style livelier.

\subsubsection{Special Editions}

The School of Mandarin Duck and Butterfly focused to attracting readers on the use of special editions, such as Violet, Non-War, Summer Living, Bi Yihong Memorial, Movies, Love, Breast Liberation Movement, Beauty Photography etc. Saturday published supplementary issues such as Weird. Zhou Shoujuan published Love in which the illustration outline was designed as double circular and cover was painted a cupid in the 115th period of resumed Saturday (Zhou, 1928). Thereafter special editions became marketing means of Saturday school Journals (Li, 2003) such as New Year, Summer Living, Mid-Autumn, Youth Depression, Love, Lover, Divorce, Virgin Psyche, Patriotic, Nation Humiliation etc.

\subsubsection{Special Issues}

The School of Mandarin Duck and Butterfly was good at editing special issues, such as ones opened up by Zhou Shoujuan when editing Free Talk including special issues of festivals and memorial days, the fiction special issues, family special issues and occasional special issues. Establishment of special issues, as Zhou Shoujuan's innovation, made content of Free Talk more systematic and increase reader's interest (Yin, 2009). In addition, the School of Mandarin Duck and Butterfly published many magazines for film companies, such as the authors of special issues of Stars of Stars Company were from the School of Mandarin Duck and Butterfly. It uses film name as title of each period the articles in which involved film criticism, news, pictures, subtitles and original story.

\subsubsection{Illustrations with Pictures and Essays}

The School of Mandarin Duck and Butterfly carried forward the popular style Illustrations with Pictures and Essays of novel magazines in the late Qing Dynasty. Saturday published about 600 pictures in the early and late 200 periods among which there published important people such as Sun Wen and his wife,Liang Qichao, Wu Zhihui, Wu Peifu, Cao Kun and the others as well as photos of some literary people such as Lin Qinnan, Mei Lanfang and western novelists such as Dante, Maupassant, Mark Twain, Shakespeare, Tolstoy, Dickens, haggard etc. In addition, it also published news photos such as was three marshals of EFR coalition, life of European captives, vehicles of Europe Red Cross in battlefield, German torpedo boats, Belgium armored motor vehicles cars in 1915 when European war was intense. In addition, it also published photos matching Sherlock Holmes's detective stories adapting to popular detective novels at that time. The content of copperplate pictures in its late 100 periods was more abundant than its early 100 periods which included historic scenic, western paintings and all kinds of characters. There were many photos of the School of Mandarin Duck and Butterfly such as Zhou Shoujuan, Yuan Hanyun, Tian Xuwosheng, Xie Zhiguang, Jiang Xiaojian, Chen Xiaodie etc. New novel was illustrated with photos in each period such as photos of world famous writers and actors, such as photos of 
Russian novelist Tolstoy published in inaugural issue in 1902, English famous writer William Byron and French big writer Hugo published in the second period. Month, Novels Forest published pictures of landscape, characters, event in the column Picture. Violet initiatively set up 20-open format in pursuit of fashionable look and modeled magazines in Europe and the United States, inserted pattern painting and painting of beautiful woman, which incited reader and improved publication sales.

\section{Publishing and Marketing Creativity}

\subsection{Positioning Creative}

The School of Mandarin Duck and Butterfly focused on positioning when engaged in publishing activities. The first is weekly Positioning. Weekly is a weapon of disseminating knowledge and advocating ideas at the cultural development period (Song, 2006). Saturday competed with rivals through prominent characteristics of publishing on Saturday, rare in press at the time ( $\mathrm{Jia}, 2005$ ). The second is color positioning. As the representative of journals, Red Rose focusing on social secularization could be called the red series; As the representative of journals, Violet reflecting more directly city life could be called the purple series; As the representative of periodicals for which celebrity authors worked who cared more about politics problems, Coral could be called the color series(Liu.2006:58-63). The third is magazine group positioning. The School of Mandarin Duck and Butterfly founded a series of publications relying on important magazines and formed magazine group, such as Saturday, Half Month and Violet edited by Zhou Shoujuan. Formation of magazine group which satisfied citizens' leisure psychology marked shaping of publishing industry group.

\subsection{Propaganda Creativity}

The School of Mandarin Duck and Butterfly promoted their magazine by a variety of forms, such as advertising language often appeared in Free Talk edited by Zhou Shoujuan(Chun,1923). News fixed position for advertisements such as advertising of Game World: a rose road out of boring life among which there were sweet novels, thick conversation, strange notes and vivid games. Saturday also attracted readers through poetry advertising such as poem advertising in the sixth period. Its advertising design also focused on innovation such as advertising in Shanghai saying which was full of kindness and substantial to public readers (Liu, 2006). The column Publishing News regularly introduce Saturday, Violet and other publications in Shen Daily Free Talk.The School of Mandarin Duck and Butterfly was also good at recommending and propagating each other in colleague magazines, such as Week edited by Bao Tianxiao advanced "Director Bao Tianxiao" as signs in Half Moon and told readers time of publishing, characteristics, retail price etc; Grand Sight on Novels publishing advertisement of Women, Times as well as its supplement Sideshow Story; Novel Pictorial publishing advertisement of Grand Sight on Novels; Half Month, Game World, Week carrying with interactive advertising(Shen,2006).

\subsection{Promotion Creativity}

Characteristic style of literary creation of the School of Mandarin Duck and Butterfly favored brand promotion for booksellers, such as Zhou Shoujuan's romance novels Promise, Blood, Record of Gramophone, Father and Son, Ten-Year Widowhood, Feet etc popular with readers. Therefore the School of Mandarin Duck and Butterfly became brand of promotion. Zhou Shoujuan was called "Love Guru" by the booksellers (Wang, 1924). World Press specially invited Yan Duhe, Bu Xiaosheng, Jiang Hongjiao, Shi Jiqun, Wang Xishen, Cheng Xiaoqing, Li Hanqiu etc to write romance novels. Bao Tianxiao tried various ways to inspire readers' purchase desire, improved journals sales, Strengthened interaction between publication and audience and encouraged readers to actively participate in when editing various publications. Times had released reward for novel creation. Women Times also hosted reward activities of which the theme was the reason for women's participation in politics. In addition, the School of Mandarin Duck and Butterfly developed sales channels and established stable sales networks. Bao Tianxiao arranged people to paste the bills in the countryside, sold and consigned newspaper through small grocery store around towns in Suzhou when founding Vernacular newspaper of Suzhou. He also established distribution network radiating to the small towns in big and medium city through the relevant newspapers, bookstores and agencies relying on metropolitan advantages and good interpersonal relationship after reaching Shanghai. The School of Mandarin Duck and Butterfly used movie as a way of promotion through the joint between film and literature market, such as Heart Words attracted reader's attention by photos of actresses, stars and authors, set up national stars selected conference, announced results of votes, organized criticisms on stars and excellent movies. Saturday provided a wide variety of gifts such as fine paintings on celebrities, ten thick volumes of hardcover books at half price as well as stage photos, illustrations, pictorials etc for users of half-year subscription in order to promote the market(Wei,1984). 


\section{Cross-media Industry Creativity}

Compared with traditional industry, creative industry's effect of value increment which had very strong radiation due to its high industrial relevance was more obvious. The School of Mandarin Duck and Butterfly contributed to formation of early Chinese culture creative industry chain. The commercial maximized value of industrial chain of novels and film product was produced on the use of martial novel Hero Biography of the School of Mandarin Duck and Butterfly in early Chinese culture and creative industry. On spring of 1928, Star film company of Shanghai adapted Ping Jiangbuxiaosheng's novel Hero Biography into the first episode of film Burning Temple of Red Lotus and led to a total of 28 episodes by 1931, which made martial movie crazy. Star Company brand promoted value of Burning Temple of Red Lotus which had legions of media industry chain and strong marketing advocacy. It cost Star Company a lot to shoot Burning Temple of Red Lotus. Star Company picked up famous actors very scrupulously, demanded film version to be completely faithful to the original and film to be based on the author's writing schedule, created the heart shaking visual effect on full use of technology at the time.

Creativity brought not only profit of a film or a book but related products around creative cultural phenomenon in the mature culture industry chain. Film diffusion similarly played propaganda promotion effect to the novels. Authors of martial novels became brands of film propaganda with popularity of Burning Temple of Red Lotus. The effects of martial novels such as Hero Biography were expanded greatly especially on the Youth (Si, 2000). The films became channels of driving novel sales. Comic novels adapted according to martial novels and films were very popular at the end of 1920's. Appearance of film provided more direct template for graphic novels (Wan, 2007).Comic strips appeared in large numbers with martial arts as theme after the epidemic of martial films. Six kinds of comics had been constantly published by Shanghai world bookstore since the beginning of 1927, including Three Kingdoms, Burning Temple of Red Lotus which published by Pu Yi Bookstore (Zhu, 1987).

\section{Conclusion}

The interaction between the School of Mandarin Duck and Butterfly and early Chinese publishing industry fully implied budding of early Chinese culture and creative industry and provided experiences and enlightenments, such as paying attention to content providing, actively adjusting the model of content publishing, mining creativity adapting to needs of mental and psychosocial of people. It will contribute to current Chinese development of culture creative industry through research on relations between the School of Mandarin Duck and Butterfly and early Chinese creative publishing industry based on historical data.

\section{References}

Bao, Tianxiao. (1971). Memories on Bracelet shadow chamber. Hongkong: Hongkong Dahua press.

Chen, Shan. (2008). The Origin and Formation of Thought of Chinese Film Theory. Contemporary Film, (1), 60-67.

Chun, Meng. (1923, July 2). Best Novels. Shen Daily Free Talk.

Jian, Jinli. (2005). On Editorial Thought of Saturday. Master degree of Henan University.

Li, Pin. (2003). Operation on Mass Periodicals. Beijing: China Encyclopedia press.

Li, Song. (2005). Modernity Pursuit and Reflection: The Mandarin Duck and Butterfly-Saturday School and Culture Industry. Journal of Industrial and Commercial University of Chongqing, (5), 122-125.

Liu, Tiequn. (2006). Saturday: Representative Work of Periodicals of Literature Catered to Ordinary Citizens in Early Years of The Republic of China. Journal of Guangxi Normal University, (2), 58-63.

Liu,Yangti. (1997). School in Evolution: On the School of Mandarin Duck and Butterfly. Beijing: The Publishing House of Literary Federation of China.

Nie, Chun. (2008). Bao Tianxiao and Chinese Modern Newspaper Industry. New Century Library, (1), 95-98.

Shen, Qinghui. (2006). On Bao Tianxiao and His Novels. Doctoral dissertation of East China Normal University.

Si, Nian. (2000). On Pingiiangbuxiaosheng. Century, (6), 53.

Song, Yingli. (2006). Development of History on Chinese Journals. Zhengzhou:Henan University press.

Tang, Zhesheng. (2007). On Influence of Modern Mass Media on Creation Mechanism of Modern Chinese Literature. Jiangsu Academy of Social Sciences, (5), 187-192.

Wan, Shaojun. (2007). On Chinese Comic. Painting Art, (10), 92-97. 
Wang, Dungen. (1924). History on Famous Authors in the Period of Ten Days. Social Flower, 1(12).

Wei, Shaochang. (1984). Research Data of the School of Mandarin Duck and Butterfly. Shanghai: Shanghai literature and Art Publishing House.

Yin, Zhimin. (2009). On Free Talk Chief-edited By Zhou Shoujuan. Master degree thesis of East China Normal University.

Zhou, Lirong. (2005). The School of Mandarin Duck and Butterfly and Publishing Industry in Republic of China. Publishing Historical data, (2), 84-88.

Zhou, Shoujuan. (1919, September 23). Film Talks. Shen Daily Free talk.

Zhou, Shoujuan. (1928, August 25). Memories on Saturday. Saturday.

Zhu, Honghong. (2007). On Book Binding Design. Master degree of Jiangnan University.

Zhu, Lianbao. (2008). Historical Memories on World Bookstore. Publishing Historical Data, (2), 55. 